\title{
A NOVEL CONCEPT FOR HIGH CONVERSION \\ OF COAL TO LIQUIDS
}

Progress Report No. 7

(Covering the period March 1, 1990 - May 31, 1990)

Wendell H. Wiser, Principal Investigator

Joseph Shabtai, Co-Principal Investigator
Department of Fueis Engineering
University of Utah
Salt Lake City, Utah 84112

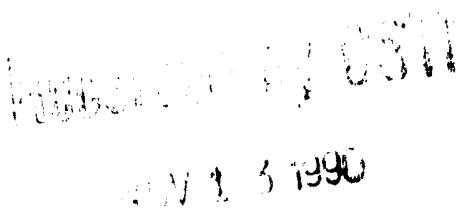

Prepared for the United States Department of Energy

under Contract No. DE-AC22-88PC88817

June, 1990

PROPRIETARY INFORMATION: The discussion contained

* on Page 2 of this report is submit.ted in confidence, therefore page? is stamped CONFIDENTIAL.

paie 2 has been deleted 


\section{PERSONNEL}

In addition to the Principal Investigators, research personne? participating in this project during the reporting period are:

Mirs. Qiuping Yang, Graduate Student

Mr. Zhaoyi Yang, Graduate Student

\section{OBJECTIVES AND SCOPE OF THE RESEARCH PROJECT}

The overall objective of this work is to demonstrate conversion of coal to produce at least $70 \%$ by weight of the coal as liquids, with ratios of 1 iquids to gases in excess of $10 / 1$, resulting in low hydrogen consumption and a significant reduction in the cost of producing hydrocarbon 1 iquid fuels from coal. Utilizing a small continusus-flow reactor designed and constructed for this research, the maximum possible ratio of liquids to gases will be defined, operating at short residence times of a few seconds, at the same time converting more than $70 \%$ of the carbon in the coal to liquids. The oractical ability to attain coal particle center-line temperatures of $500{ }^{\circ} \mathrm{C}$ in one second or less, using hot hydrogen gas in turbulent flow, will also be demonstrated. Particle heat-up rates for a few selected system pressures and particle sizes will be determined. Catalysts will be screened and selected for sufficient activity and selectivity to achieve the desired yields, with potential for operation within satisfactory economic boundaries as to cost, recoverability and regeneration. Particle heat-up rates for small coal particles slurried in a super-critical hydrocarbon-type fluid will be examined, using hot hydrogen gas in turbulent flow as the heat transfer medium.

\section{DISCLAIMER}


specified. Following delivery of the controller, a series of experiments revealed that the response time of the instrument was too slow to permit us to reach our objective. We were unable to obtain from this vendor, or any other vendor, an assurance that any commercially available controller cound meet our needs. This explains why it became necessary for us to design and construct our own temperature controller.

Following is a description of the important features of the temperature controller designed by Dr. K. S. Yang, a post-doctoral Research Associate in our Program.

A. The Controller Circuit.

1. Signal input stage. Because of the extremely small signal (about $0.04 \mathrm{mV} /{ }^{\circ} \mathrm{C}$ for a $\mathrm{K}$-type thermocouple), the input stage is designed to provide an imput resistance of about $10^{12}$ ohms (the bias current is about 1 to $2 \mathrm{nA}$ ). To reject foreign noise and power switch disturbances, differential inputs and feedbacks are extensively used in the design of the input and subsequent amplifier stages.

2. Amplifier and comparator stages. The thermocouple signal from the input stage is first amplified 100 times and compared with the setting point. The voltage difference is amplified another 100 times. The resulting output is then compared with $2.5 \mathrm{~V}$. The output from the last comparator is TTL compatible. The gain of this stage is 1000. A small hysteresis 10op of several $\mathrm{mV}$ is applied to speed the output transition and eliminate possible circuit oscillations due to the high gain and stray capacitance, which is a very cormon problem associated with wire-wrapping circuits.

3. Logic Circuit. The logic circuit is implemented to control (1) power relays, (2) timing circuit, and (3) panel displays. 
4. Power supply. A 12-volt automobile battery is used as the main power supply. A regulating circuit was designed to provide a voltage of 5 volts for the logic and part of the analog control circuit. The power output to the reactor is controlled by a 3-ohm variable resistor placed in series with the heating screen of the reactor. The maximum power output to the reactor is about 1000 watts.

B. Testing Results.

1. Temperature reading error: within $\pm 1^{\circ} \mathrm{C}$.

2. Sensitivity: $\pm 2{ }^{\circ} \mathrm{C}$ (or $8 \times 10^{-5} \mathrm{~V}$ )

3. Maximum heating rate: $>1500{ }^{\circ} \mathrm{C}$ per second.

C. Physical Limitations of the Control System.

Response time delay appears to be the main limitation of the control system. The possible sources for the time delay are:

1. Thermocouple: $0.01 \mathrm{sec}$. (vendor's specification).

2. Control circuit propagation delay: No more than a few $\mu$ s.

3. Relays; $0.01 \mathrm{sec}$. to completely switch on and off (worst case, vendor's specification).

4. Heat transfer from screen to coal powder.

5. Heat transfer from coal powder to thermocouple. Hence, if there is no heat transfer delay, the theoretical maximum overshooting is about $10{ }^{\circ} \mathrm{C}$ at a heating rate of $500{ }^{\circ} \mathrm{C} / \mathrm{sec}$, as a. result of $0.02 \mathrm{sec}$ response delay. The subsequent vibration around the setting point should be about $\pm 8{ }^{\circ} \mathrm{C}$ with a period of about 0.05 sec. However, the testi, ; results indicated that the total time delay could be as high as 0.1 to $0.2 \mathrm{sec}$. To avoid the overshooting problem, the heating rate must be lowered so that the rate of heat 
loss from the screen and coal sample at the setting point is about the same as that of heating.

The portion of the work to use this temperature controller is being performed by Mrs. Qiuping Yang, one of the gradlate students.

Work is continuing in the deveopment of a continuous flow reactor system. The reactor system components which were retained from prior experimental work, together with other components purchased for this work which have arrived, have all been assembled into the continuous flow system. There are still two key components which have not yet been dei ivered by the vendors, although the delays have reached several months. The absence of these components has prevented us from testing the full reactor system. These delays are very frustrating. We are finding that an increasing number of manufacturers of instruments and reactor components do not maintain a shelf inventory of many of the products listed in their catalogs. Hence after they receive an order they proceed to manufacture the item requested in the order. The explanation given after receiving a complaint is that the amount of capital which would be "tied up" in inventory would be too great, and more than they could accomodate.

The major task of identifying all of the circuitry of the rather elaborate instrument control panel, as mentioned in the prior report, has been completed. As soon as the remaining commonents for the system are received from the vendors, shakedown testing of the continuous reactor system wi11 commence.

This portion of the work is being performed by Mr. Zhaoyi Yang, one of the graduate students. 

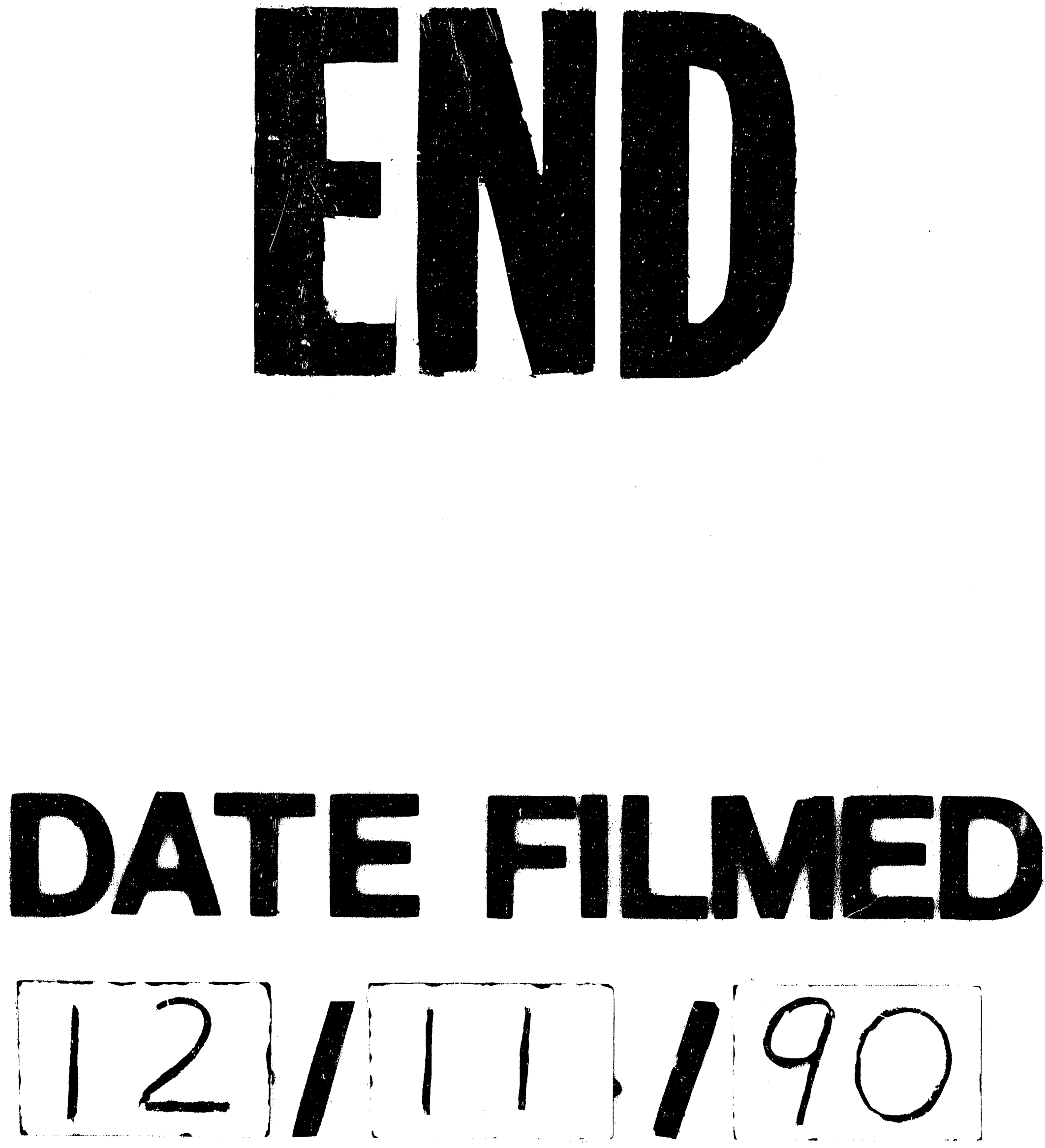
